Case Report

\title{
Primary Pulmonary Malignant Melanoma: Report of an Important Entity and Literature Review
}

\author{
Christos Kyriakopoulos, ${ }^{1}$ George Zarkavelis, ${ }^{2,3}$ Artemis Andrianopoulou, ${ }^{4}$ Alexandra \\ Papoudou-Bai, ${ }^{5}$ Dimitrios Stefanou, ${ }^{5}$ Stergios Boussios, ${ }^{2,3}$ and George Pentheroudakis ${ }^{2,3}$ \\ ${ }^{1}$ Department of Pulmonary Medicine, Medical School, University of Ioannina, Stavros Niarchos Avenue, 45110 Ioannina, Greece \\ ${ }^{2}$ Department of Medical Oncology, Medical School, University of Ioannina, Stavros Niarchos Avenue, 45110 Ioannina, Greece \\ ${ }^{3}$ Society for Study of the Clonal Heterogeneity of Neoplasia, Ioannina, Greece \\ ${ }^{4}$ Department of Radiology, Medical School, University of Ioannina, Stavros Niarchos Avenue, 45110 Ioannina, Greece \\ ${ }^{5}$ Department of Pathology, Medical School, University of Ioannina, Stavros Niarchos Avenue, 45110 Ioannina, Greece
}

Correspondence should be addressed to Stergios Boussios; stergiosboussios@gmail.com

Received 6 December 2016; Revised 12 February 2017; Accepted 14 February 2017; Published 2 March 2017

Academic Editor: Jose I. Mayordomo

Copyright (C) 2017 Christos Kyriakopoulos et al. This is an open access article distributed under the Creative Commons Attribution License, which permits unrestricted use, distribution, and reproduction in any medium, provided the original work is properly cited.

Malignant melanoma involving the respiratory tract is nearly always metastatic in origin, and primary tumors are extremely rare. Published data on primary pulmonary malignant melanomas are limited. Up to now 40 relevant cases have been reported in the English literature. Herein, we report a case of a 56-year-old female patient who presented with intracranial metastases due to primary pulmonary melanoma. She underwent bronchoscopy and died 5 months after the initial diagnosis despite the administered biochemotherapy and subsequent immunotherapy. To establish the diagnosis of primary pulmonary malignant melanoma, any extrapulmonary origin was excluded by detailed examination and radiographic imaging. Moreover, an extensive review of the literature regarding this rare entity has been performed.

\section{Introduction}

Worldwide, approximately 160,000 new cases of melanoma are diagnosed each year, and about 41,000 melanoma-related deaths occur annually $[1,2]$. Melanoma is a malignant neoplasm of melanocytes, and more than $90 \%$ of the reported cases are cutaneous in origin. Although malignant melanoma mainly occurs on the skin, it has also been described in other mucosal sites and organs, including the oral cavity, paranasal sinuses, esophagus, larynx, vagina, anorectal region, and liver $[2,3]$. Approximately $5-10 \%$ of patients with metastatic melanoma have a primary melanoma of unknown origin $[2$, 4]. Primary malignant melanoma of the lung is an extremely rare nonepithelial neoplasm that accounts for only $0.01 \%$ of all primary lung tumors $[1,5]$.

To date, 40 cases have been reported in the English literature [6-41]. Patients' characteristics and demographics are depicted in Table 1. The mean age at diagnosis is 59.1 years (range 29 to 90) with male prevalence. Melanomas involving the lung are almost always metastatic, and it is extremely rare to find a true primary lesion. Metastasis from an occult primary lesion must be excluded by proposed criteria $[3,4$, 31].

\section{Case Presentation}

A 56-year-old female without comorbidities and a 50-packyear smoking history was referred to the emergency department with a severe right frontal headache. The onset was abrupt and the preceding symptoms and signs included nausea and ataxic gait. Neither cranial nerve abnormalities nor focal limb weakness was spared. Initial laboratory values were within the reference range. The patient underwent a magnetic resonance imaging (MRI) which revealed multiple nodular lesions throughout both cerebral and cerebellar hemispheres with an unusual lamellated pattern of 


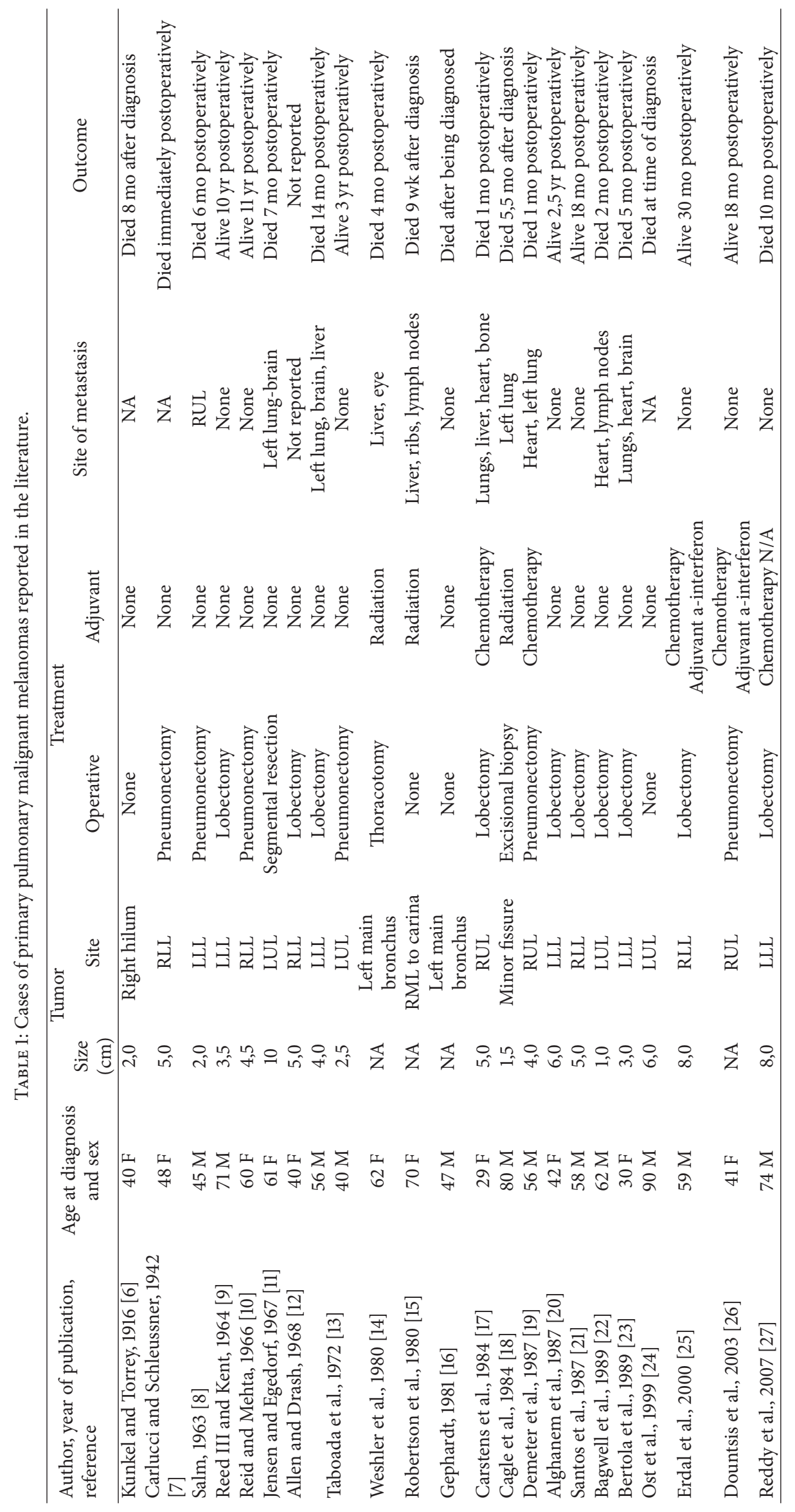




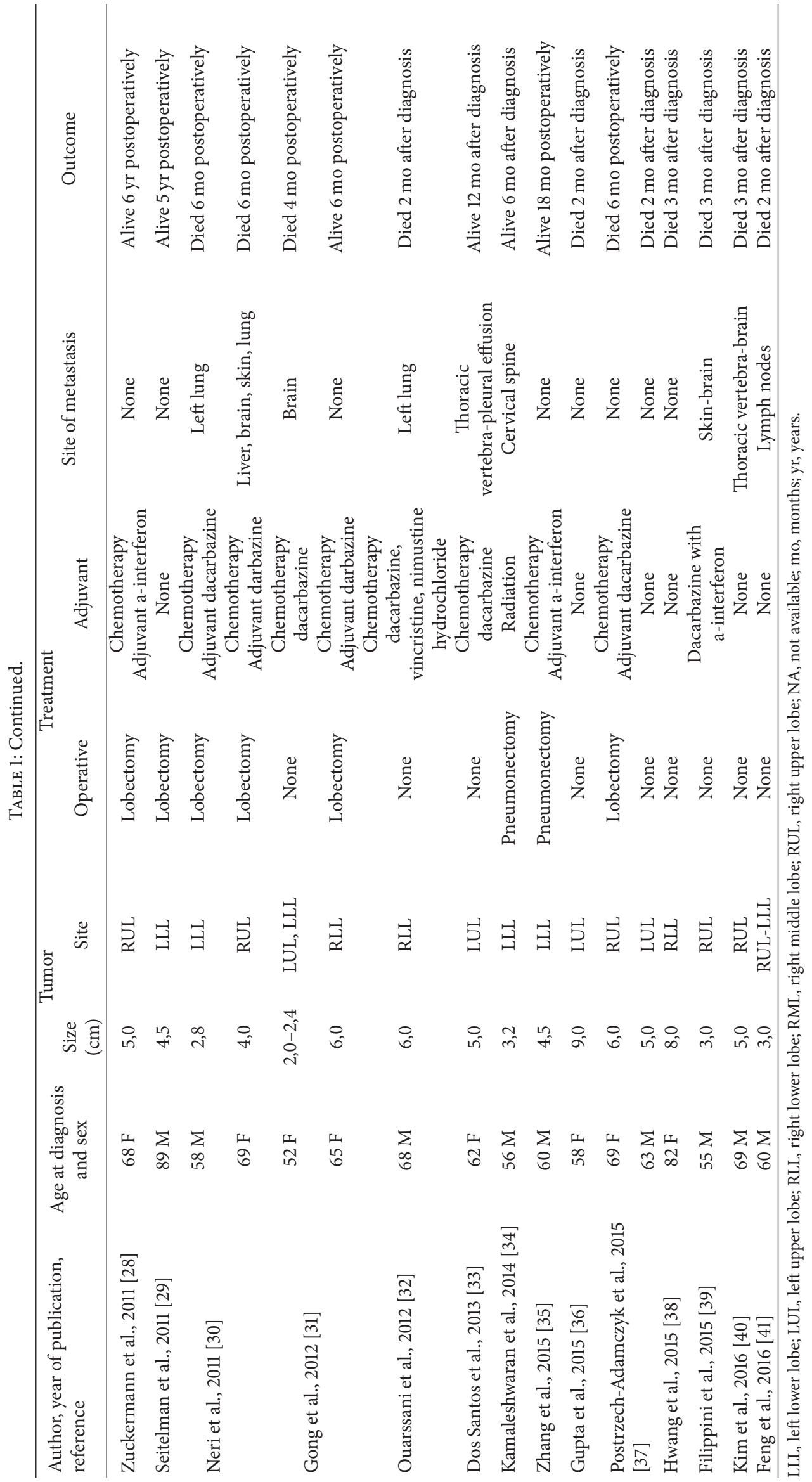


enhancement. The demonstrated significant vasogenic edema did not led to midline-shift (Figures $1(\mathrm{a})-1(\mathrm{~d})$ ). Chest Xray detected opacity with a lobulated contour in the right upper lobe (Figure 1(e)). A contrast-enhanced chest computed tomography (CT) showed an ill-defined mass in the anterior segment of the right upper lobe and right hilar lymphadenopathy (Figure 1(f)). No additional abnormalities were observed on subsequent detailed metastatic work-up including abdominal CT and whole body bone scintigraphy. Due to the neurological symptoms, the patient underwent whole-brain radiotherapy (WBRT). Fractionation of $3 \mathrm{~Gy}$ per day was used, reaching a total dose of $30 \mathrm{~Gy}$ in two weeks; in addition, the patient received intravenously dexamethasone during WBRT. The performed bronchoscopy detected a bulging lesion located at the membranous portion of bronchus of the anterior segment of the right upper lobe. The lesion did not appear to be covered with normal bronchial mucosa. Subsequently, a biopsy was performed (Figure 2), and the histopathology showed infiltration by atypical melanocytes containing melanin pigmentation (Figures 3(a) and 3(b)). Positive immunohistochemical staining of MART-1, S-100 protein, and HMB-45 and lack of expression of cytokeratin (CK), epithelial membrane antigen (EMA), synaptophysin (Syn), and high molecular weight cytokeratin confirmed the diagnosis of pulmonary melanoma. Molecular profiling was conducted which failed to detect any mutations.

To rule out the diagnosis of melanoma metastasis from a primary site, an extensive skin and fundoscopic examination was carried out. Endoscopy of both upper and lower gastrointestinal tract as well as cystoscopy did not detect any additional abnormalities. Indeed, a panendoscopy of the upper airway failed to demonstrate extrapulmonary disease. Based upon the histological characteristics, immunohistochemical features, and clinical data, the diagnosis of primary pulmonary malignant melanoma was established.

Surgical resection of the lung mass was not indicated due to the brain metastases. The completion of WBRT resulted in transient remission of the neurological symptoms and the patient was switched to dacarbazine (DTIC) in combination with recombinant interferon alpha-2a (rIFN- $\alpha-2 a)$. Due to unacceptable toxicity rIFN- $\alpha-2 \mathrm{a}$ was discontinued three weeks after being administered.

The treatment did not provide any clinical benefit; instead patient's condition deteriorated and ipilimumab was added to DTIC. The evaluation of response was planned to be performed after four cycles of the administered combination therapy. Indeed, the patient was admitted to the hospital with progressive exertional dyspnoea and persistent chest tightness. She died five months after the initial diagnosis. Massive pulmonary embolism was determined as the cause of death in the performed autopsy.

\section{Discussion}

Published data on primary pulmonary malignant melanoma are limited. The performed systematic review confirms that only 40 cases appeared in the literature since 1916 (Table 1) [6-41]. Median age of presentation was 59.1 years (range,
29 to 90 ). The gender distribution for the entire population was 21 males to 19 females. Therapeutic management is a great challenge, and clinical trials are clearly warranted to evaluate the efficacy of local and systemic adjuvant treatments in decreasing recurrence and improving survival. Surgical resection was the primary treatment (27 out of the 40 reported patients, 67.5\%) while adjuvant systemic treatment was delivered just to 19 patients (47,5\%). In addition, palliative radiotherapy to distant sites had been administered in 4 patients $(10 \%)$. In general, it seems that most patients (19 out of 40) were diagnosed with metastatic disease (47,5\%). The sites of metastatic involvement included the contralateral lung, liver, brain, bones, and pericardium. The outcome was very dismal and the majority (26 out of 40 patients) survived less than 18 months (65\%).

Primary pulmonary malignant melanoma resembles that of the skin or mucosa. A diagnosis of primary malignant melanoma of the lung is based on clinical and pathological criteria $[4,26,42]$. The clinical criteria include absence of either history suggestive of a previous melanoma or demonstrable melanoma outside the thorax at the time of surgery. Furthermore, the presence of a solitary lung mass or nodule is required. The established pathological criteria incorporate pathognomonic immunohistochemical staining for S-100 and HMB-45, evidence of junctional change with nesting of melanoma cells or spindle cells arranged in fascicles, and invasion of bronchial epithelium in an area without epithelial ulceration. Indeed, other melanotic tumors, such as melanotic medullary carcinoma of thyroid [43] and pigmented neuroendocrine carcinoma should be excluded [44]. In our patient, immunohistochemical staining demonstrated that the tumor cells expressed HMB45, S-100, and MART-1, whereas they did not express CK, EMA, Syn, and high molecular weight cytokeratin. Thus, the diagnosis was reliable.

The precise histogenesis of pulmonary malignant melanoma remains controversial. Most experts support that melanocytes migrate concomitantly with reduced growth of the primordial tubular respiratory tract during fetal growth. It is also suggested that these cases are a metastatic form of an antecedent skin lesion that either is unrecognized or has spontaneously regressed. Interestingly enough, melanocytes and melanocytic proliferation are present in the larynx and esophagus. Furthermore, larynx, esophagus, and lungs all share a common embryologic origin, implying the possible migration of melanocytes [35, 36, 42].

As far as therapy is concerned, patients diagnosed with primary pulmonary malignant melanoma should undergo lobectomy or pneumonectomy with lymph node dissection $[2,42,45]$. Although interferon $a-2 b(\operatorname{rIFN}-\alpha-2 b)$ is frequently offered to mucosal melanoma patients as systemic adjuvant therapy, it has not been formally studied in this patient population. Most of the patients with mucosal melanoma have micrometastases at the time of diagnosis of the primary tumor. However, adjuvant therapy has not been studied in a randomized fashion because of the rarity of the disease. The role of chemotherapy is not fully clarified. Biochemotherapy which consists of combination of chemotherapy and immunotherapy is an acceptable choice 


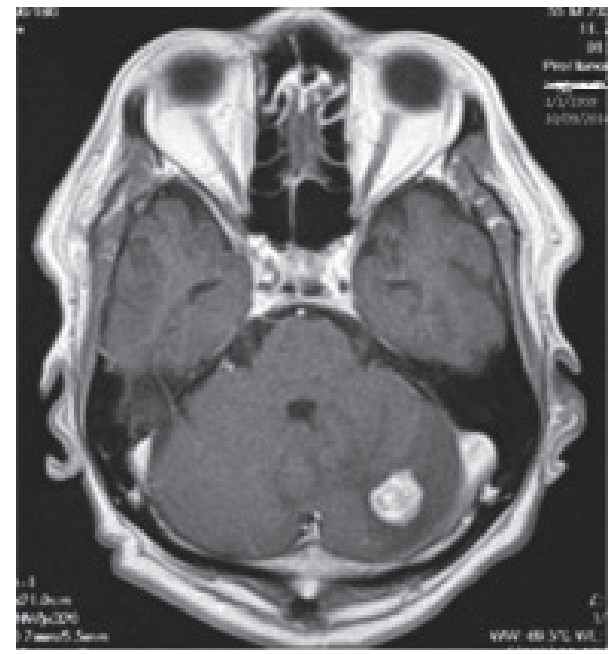

(a)

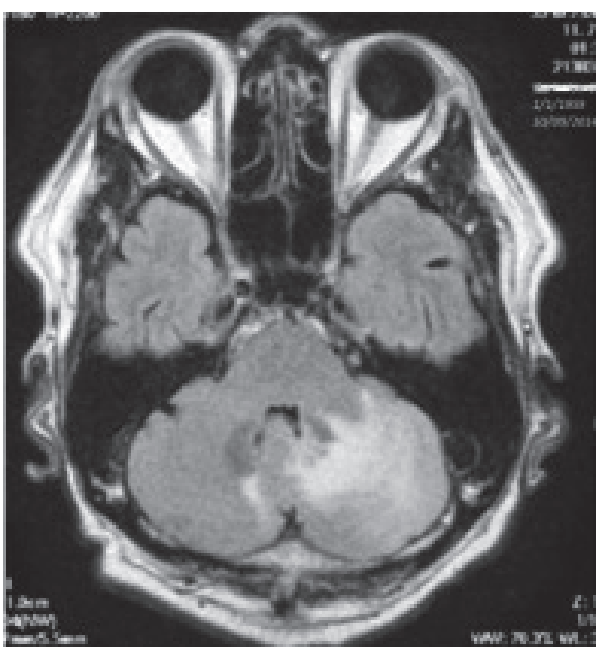

(c)

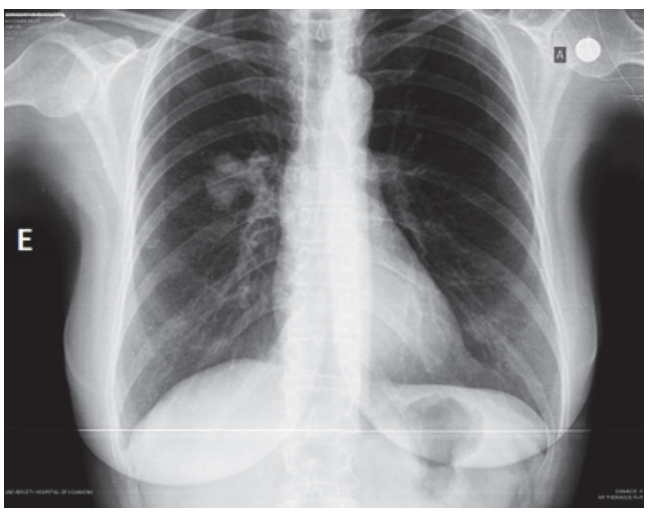

(e)

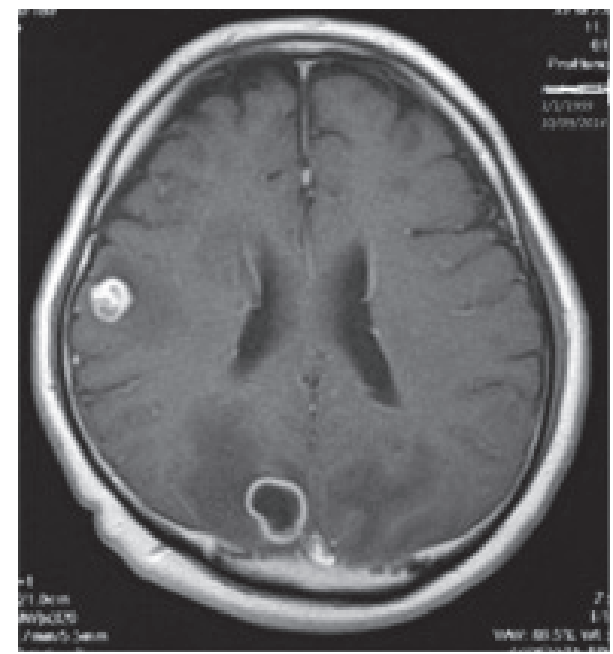

(b)

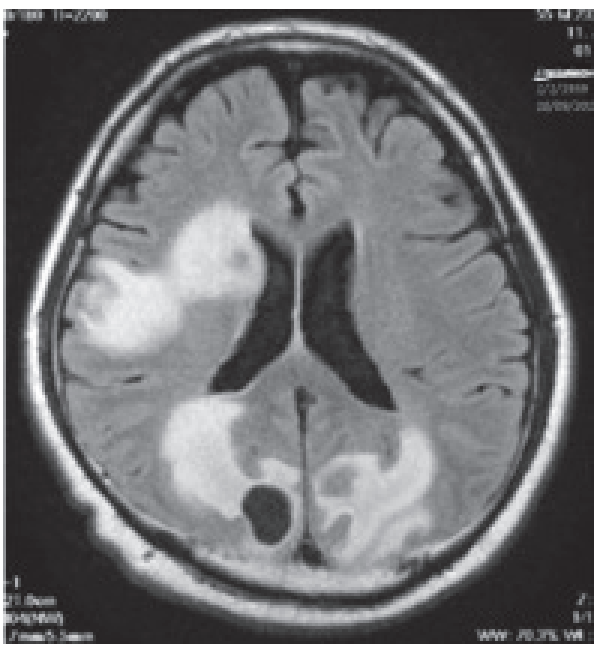

(d)

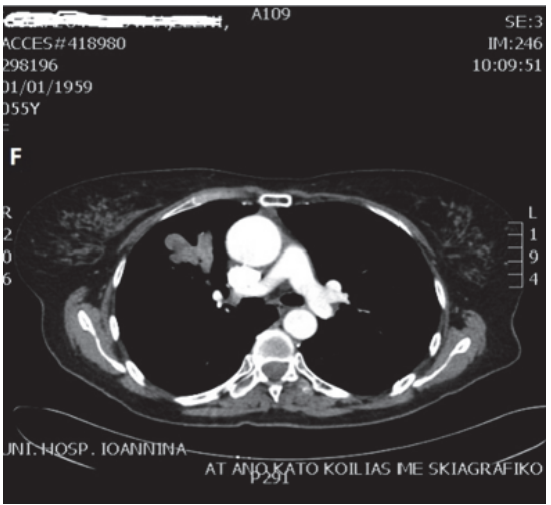

(f)

FIgure 1: ((a) and (b)) Postgadolinium T1-weighted MR images demonstrate multiple enhancing nodular lesions throughout both cerebral and cerebellar hemispheres, several of which have an unusual lamellated pattern of enhancement. ((c) and (d)) T2-weighted and FLAIR MR images with paired postgadolinium T1-weighted images demonstrate significant vasogenic edema associated with several of these lesions. (e) Chest X-ray showing tumor in the anterior segment of right upper lobe. (f) Chest CT showing tumor in the anterior segment of right upper lobe with ill-defined shape and right hilum lymph nodes. 


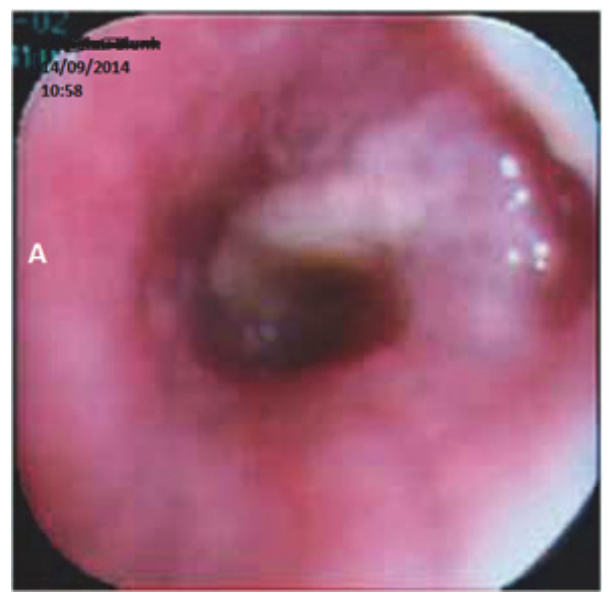

FIgURE 2: Bronchoscopic examination showed a bulging lesion in the bronchus of the anterior segment of the right upper lobe.

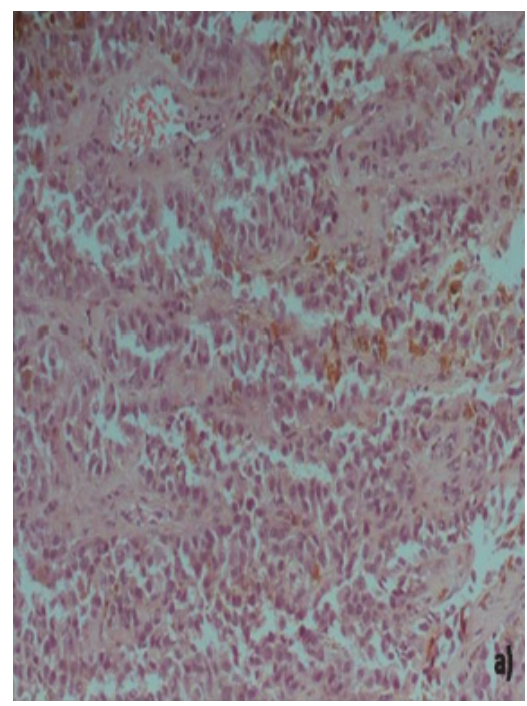

(a)

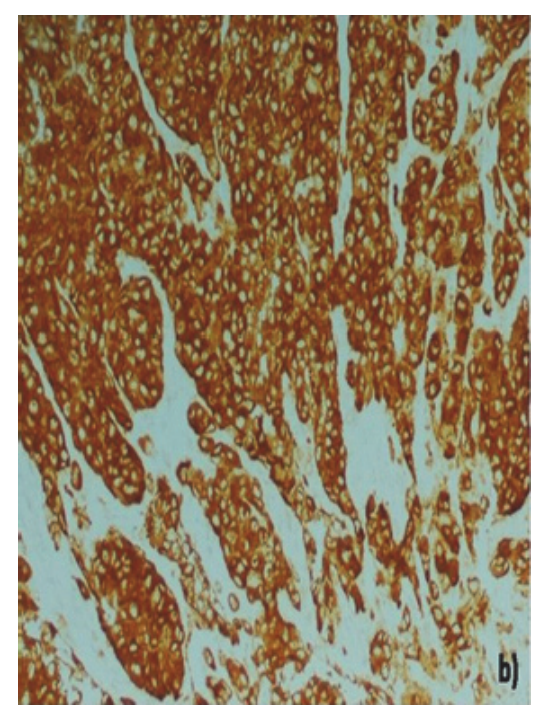

(b)

FIGURE 3: (a) Bronchial biopsy with infiltration by atypical melanocytes containing melanin pigmentation (H\&E, magnification $\times 200)$. (b) Immunohistochemical expression of Melan A in neoplastic cells $(D A B$, magnification $\times 200)$.

after always taking into consideration patients' performance status and comorbidities, as well as the relatively expanded toxicity profile of the combination therapy. When aggressive bulky disease is documented, biochemotherapy may be initiated with close monitoring of the patient. Disease stabilization or partial responses have been documented in the cost of toxicity. Our patient did not tolerate well rIFN$\alpha$-2a combined with DTIC, mandating administration of ipilimumab instead. It seems that overall survival benefit has not been demonstrated in metastatic setting [46]. The reported patient achieved overall survival of 5 months which is in accordance with the literature.

The discovery of activating mutations in the serine/threonine kinase BRAF in approximately $50 \%$ of all melanomas led to the Food and Drug Administration (FDA) approval of the BRAF inhibitor vemurafenib in 2011 [47]. Dabrafenib gained FDA approval in 2013, not only for the treatment of BRAF-V600E expressing melanomas, but also for those expressing BRAF-V600K [48]. In the same year, trametinib, the first MEK inhibitor marketed for cancer treatment, was approved by the FDA [49]. Moreover, MEK inhibitors acting in the down signaling RAS pathway can be used preferably in combination with a BRAF inhibitor. Typically, disease remission can be established within weeks, although resistance to therapy is observed usually after a median of 6 months.

For BRAF wild type metastatic melanoma immunotherapy with the anticytotoxic T-lymphocyte antigen-4 (CTLA4) antibody ipilimumab may be the first choice for treatment. Ipilimumab activates the $\mathrm{T}$ mediated immune response against the tumor cells which have the ability of immune evasion. Response is achieved late after the therapy, but it can be durable. Finally, in case where c-Kit mutation is present, mainly observed in mucosal or acral melanoma, 
the BCR-ABL tyrosine kinase inhibitor imatinib has shown some effect but not durable $[26,50]$. In our patient BRAF, NRAS, and c-KIT testing was negative for mutations and the multidisciplinary group decided to initiate biochemotherapy.

Recently immunotherapy with programmed death-1 (PD-1) checkpoint inhibitors has raised much attention since both nivolumab and pembrolizumab have been approved by the FDA in 2014 for the treatment of metastatic cutaneous melanoma presenting with excellent risk profile [51]. Even though several new agents have been approved for the treatment of cutaneous melanoma, including the combination of nivolumab and ipilimumab, there is a paucity of published information regarding the efficacy and safety of this combination in other melanoma subtypes. Nivolumab may be effective in mucosal melanoma regardless of the tumor molecular profile, similar to the demonstrated efficacy of nivolumab in cutaneous melanoma regardless of BRAF mutation status. A pooled analysis of data from six clinical studies regarding anti-PD-1 therapy in mucosal melanoma revealed that despite differences in the proportion of patients with tumor PD-L1 expression $\geq 5 \%$, overall response rate was similar between subtypes for nivolumab monotherapy and combination therapy. In contrast, lower activity in mucosal melanoma was observed across treatment groups for patients with tumor PD-L1 expression < 5\% [52]. Immunotherapy was not the first choice for our patient due to the long period needed to manifest any disease control. Unfortunately the patient died due to massive pulmonary embolism and the response to the immunotherapy cannot be assessed.

Palliative radiation therapy is utilized when bulky metastatic disease is present. Irradiation of the brain is a choice when multiple brain lesions are evident on brain imaging. This was the case in our patient. WBRT has a palliative intent whereas stereotactic radiosurgery can be applied when a restricted number of metastases are observed in central nervous system [46].

\section{Conclusion}

In this report, we added a new case of primary pulmonary malignant melanoma bringing the total number up to 41 patients in the frame of English literature. Overall, this is the rarest type of visceral melanoma and accurate diagnosis requires detailed investigation and fulfilment of specific criteria. It can appear as either a single pulmonary mass or metastatic disseminated disease. Lobectomy or pneumonectomy with lymph node dissection is the gold standard for local treatment. With the new era in melanoma therapeutics there is optimism for longer periods of survival. Due to the rarity of primary pulmonary malignant melanomas, randomized trials for assessing the relative benefits of different treatment modalities are extremely challenging.

\section{Consent}

Written informed consent was obtained from the patient prior to publication.

\section{Competing Interests}

The authors declare no conflict of interests.

\section{Authors' Contributions}

Christos Kyriakopoulos and George Zarkavelis contributed equally to this work.

\section{References}

[1] D. M. Parkin, F. Bray, J. Ferlay, and P. Pisani, "Global cancer statistics, 2002," Ca-A Cancer Journal for Clinicians, vol. 55, no. 2, pp. 74-108, 2005.

[2] S. N. Markovic, L. A. Erickson, R. D. Rao et al., "Malignant melanoma in the 21st century, part 1: epidemiology, risk factors, screening, prevention, and diagnosis," Mayo Clinic Proceedings, vol. 82, no. 3, pp. 364-380, 2007.

[3] L. Gong, Y.-H. Li, J.-Y. Zhao, X.-X. Wang, S.-J. Zhu, and W. Zhang, "Primary malignant melanoma of the liver: a case report," World Journal of Gastroenterology, vol. 14, no. 31, pp. 4968-4971, 2008.

[4] K. A. Katz, E. Jonasch, F. S. Hodi et al., "Melanoma of unknown primary: experience at Massachusetts General Hospital and Dana-Farber Cancer Institute," Melanoma Research, vol. 15, no. 1, pp. 77-82, 2005.

[5] A. F. Ülger, E. Şen, S. Erekul, and U. Gönüllü, "Malignant melanoma of the lung: is it easy to determine its origin?" Archivos de Bronconeumologia, vol. 41, no. 2, pp. 102-104, 2005.

[6] O. F. Kunkel and E. Torrey, "Report of a case of primary melanotic sarcoma of lung presenting difficulties in differentiating from tuberculosis," New York State Journal of Medicine, vol. 16, pp. 198-201, 1916.

[7] G. A. Carlucci and R. C. Schleussner, "Primary (?) melanoma of the lung: a case report," The Journal of Thoracic Surgery, vol. 11, pp. 643-649, 1941-1942.

[8] R. Salm, "A primary malignant melanoma of the bronchus," The Journal of pathology and bacteriology, vol. 85, pp. 121-126, 1963.

[9] R. J. Reed III and E. M. Kent, "Solitary pulmonary melanomas: two case reports," The Journal of Thoracic and Cardiovascular Surgery, vol. 48, pp. 226-231, 1964.

[10] J. D. Reid and V. T. Mehta, "Melanoma of the lower respiratory tract," Cancer, vol. 19, no. 5, pp. 627-631, 1966.

[11] O. A. Jensen and J. Egedorf, "Primary malignant melanoma of the lung," Scandinavian Journal of Respiratory Diseases, vol. 48, no. 2, pp. 127-135, 1967.

[12] M. S. Allen and E. C. Drash, "Primary melanoma of the lung," Cancer, vol. 21, no. 1, pp. 154-159, 1968.

[13] C. F. Taboada, J. D. McMurray, R. A. Jordan, and W. D. Seybold, "Primary melanoma of the lung," Chest, vol. 62, no. 5, pp. 629631, 1972.

[14] Z. Weshler, A. Sulkes, J. Kopolovitch, A. Leviatan, and E. Shifrin, "Bronchial malignant melanoma," Journal of Surgical Oncology, vol. 15, no. 3, pp. 243-248, 1980.

[15] A. J. Robertson, D. J. M. Sinclair, P. P. Sutton, and W. Guthrie, "Primary melanocarcinoma of the lower respiratory tract," Thorax, vol. 35, no. 2, pp. 158-159, 1980.

[16] G. N. Gephardt, "Malignant melanoma of the bronchus," Human Pathology, vol. 12, no. 7, pp. 671-673, 1981. 
[17] P. H. B. Carstens, J. G. Kuhns, and C. Ghazi, "Primary malignant melanomas of the lung and adrenal," Human Pathology, vol. 15, no. 10, pp. 910-914, 1984.

[18] P. Cagle, M. L. Mace, D. M. Judge, R. B. Teague, R. K. Wilson, and S. D. Greenberg, "Pulmonary melanoma. Primary vs metastatic," Chest, vol. 85, no. 1, pp. 125-126, 1984.

[19] S. L. Demeter, C. Fuenning, and J. B. Miller, "Primary malignant melanoma of the lower respiratory tract: endoscopic identification," Cleveland Clinic Journal of Medicine, vol. 54, no. 4, pp. 305-308, 1987.

[20] A. A. Alghanem, J. Mehan, and A. A. Hassan, "Primary malignant melanoma of the lung," Journal of Surgical Oncology, vol. 34, no. 2, pp. 109-112, 1987.

[21] F. Santos, L. M. Entrenas, F. Sebastian et al., "Primary bronchopulmonary malignant melanoma: case report," Scandinavian Cardiovascular Journal, vol. 21, no. 2, pp. 187-189, 1987.

[22] S. P. Bagwell, S. D. Flynn, P. M. Cox, and J. A. Davison, "Primary malignant melanoma of the lung," American Review of Respiratory Disease, vol. 139, no. 6, pp. 1543-1547, 1989.

[23] G. Bertola, B. Pasquotti, S. Morassut, S. Massarut, R. Sigon, and C. Rossi, "Primary lung melanoma," Italian Journal of Surgical Sciences, vol. 19, no. 2, pp. 187-189, 1989.

[24] D. Ost, C. Joseph, H. Sogoloff, and G. Menezes, "Primary pulmonary melanoma: case report and literature review," Mayo Clinic Proceedings, vol. 74, no. 1, pp. 62-66, 1999.

[25] N. B. Erdal, Z. Karakurt, I. Pandul, and C. Tahaoğlu, "A case report: primary pulmonary melanoma," Turkish Respiratory Journal, vol. 1, no. 1, pp. 72-74, 2000.

[26] A. Dountsis, C. Zisis, E. Karagianni, and J. Dahabreh, "Primary malignant melanoma of the lung: a case report," World Journal of Surgical Oncology, vol. 1, article no. 26, 2003.

[27] V. S. Reddy, J. Mykytenko, L. I. Giltman, and K. A. Mansour, "Primary malignant melanoma of the lung: review of literature and report of a case," American Surgeon, vol. 73, no. 3, pp. 287289, 2007.

[28] B. Zuckermann, M. Papiashvilli, and I. Bar, "Primary pulmonary malignant melanoma of right upper lobe of lung," Israel Medical Association Journal, vol. 13, no. 7, pp. 440-441, 2011.

[29] E. Seitelman, P. Donenfeld, K. Kay, K. Takabe, S. Andaz, and S. Fox, "Successful treatment of primary pulmonary melanoma," Journal of Thoracic Disease, vol. 3, no. 3, pp. 207-208, 2011.

[30] S. Neri, T. Komatsu, J. Kitamura, K. Otsuka, N. Katakami, and Y. Takahashi, "Malignant melanoma of the lung: report of two cases," Annals of Thoracic and Cardiovascular Surgery, vol. 17, no. 2, pp. 170-173, 2011.

[31] L. Gong, X. Liu, W. Zhang et al., "Primary pulmonary malignant melanoma: a clinicopathologic study of two cases," Diagnostic Pathology, vol. 7, no. 1, article 123, 2012.

[32] A. Ouarssani, F. Atoini, R. Reda, F. A. Lhou, and M. I. Rguibi, "Malignant melanoma of the lung: a case report," Pan African Medical Journal, vol. 11, article no. 68, 2012.

[33] C. L. Dos Santos, L. R. Fernandes, M. Meruje, and F. Barata, "Primary pulmonary melanoma: the unexpected tumour," BMJ Case Reports, 2013.

[34] K. Kamaleshwaran, S. Natarajan, J. Parthiban, S. Mehta, K. Radhakrishnan, and A. Shinto, "Rare case of extradural spinal metastasis from primary lung malignant melanoma detected with fluorine-18 fluorodeoxyglucose-positron emission tomography/computed tomography," Indian Journal of Nuclear Medicine, vol. 29, no. 1, pp. 57-58, 2014.
[35] X. Zhang, Y. Wang, and J. Du, "Primary malignant melanoma of left lower lobe of lung: a case report and review of the literature," Oncology Letters, vol. 10, no. 1, pp. 528-530, 2015.

[36] A. Gupta, D. Bhattacharya, S. Jain, and J. C. Suri, "Primary malignant melanoma of the lung: case report and literature review," The Indian Journal of Chest Diseases \& Allied Sciences, vol. 57, no. 3, pp. 181-184, 2015.

[37] K. Postrzech-Adamczyk, M. Chabowski, B. Głuszczyk-Ferenc et al., "Malignant melanoma of the lung: case series," Kardiochirurgia i Torakochirurgia Polska, vol. 12, no. 1, pp. 72-76, 2015.

[38] K. Hwang, K. Hwang, J. Jung et al., "Primary pulmonary malignant melanoma: an unexpected tumor," Tuberculosis and Respiratory Diseases, vol. 78, no. 3, pp. 272-275, 2015.

[39] A. Filippini, F. Zorzi, C. Bna', A. Arnaboldi, and T. Sabatini, "Dark sputum: an atypical presentation of primary pulmonary malignant melanoma," Respiratory Medicine Case Reports, vol. 15, pp. 118-120, 2015.

[40] S. R. Kim, H.-Y. Yoon, G. Y. Jin, Y. H. Choe, S. Y. Park, and Y. C. Lee, "Pulmonary malignant melanoma with distant metastasis assessed by positron emission tomography-computed tomography," Thoracic Cancer, vol. 7, no. 4, pp. 503-507, 2016.

[41] Y. Feng, J. Zhao, Q. Yang et al., "Pulmonary melanoma and "crazy paving" patterns in chest images: a case report and literature review," BMC Cancer, vol. 16, no. 1, article no. 592, 2016.

[42] R. Maeda, N. Isowa, H. Onuma, H. Miura, H. Tokuyasu, and Y. Kawasaki, "Primary malignant melanoma of the lung with rapid progression," General Thoracic and Cardiovascular Surgery, vol. 57, no. 12, pp. 671-674, 2009.

[43] K. Singh, M. C. Sharma, D. Jain, and R. Kumar, "Melanotic medullary carcinoma of thyroid-report of a rare case with brief review of literature," Diagnostic Pathology, vol. 3, no. 1, article no. 2, 2008.

[44] K. Iihara, K. Yamaguchi, Y. Fujioka, and S. Uno, "Pigmented neuroendocrine tumor of the lung, showing neuromelanin," Pathology International, vol. 52, no. 11, pp. 734-739, 2002.

[45] R. P. Petersen, S. I. Hanish, J. C. Haney et al., "Improved survival with pulmonary metastasectomy: an analysis of 1720 patients with pulmonary metastatic melanoma," Journal of Thoracic and Cardiovascular Surgery, vol. 133, no. 1, pp. 104-110.e2, 2007.

[46] R. Dummer, A. Hauschild, N. Lindenblatt, G. Pentheroudakis, and U. Keilholz, "ESMO Guidelines Committee. Cutaneous melanoma: ESMO Clinical Practice Guidelines for diagnosis, treatment and follow-up," Annals of Oncology, vol. 26, supplement 5, pp. v126-v132, 2015.

[47] G. V. Long, A. M. Menzies, A. M. Nagrial et al., "Prognostic and clinicopathologic associations of oncogenic BRAF in metastatic melanoma," Journal of Clinical Oncology, vol. 29, no. 10, pp. 1239-1246, 2011.

[48] P. A. Ascierto, D. Minor, A. Ribas et al., "Phase II trial (BREAK2) of the BRAF inhibitor dabrafenib (GSK2118436) in patients with metastatic melanoma," Journal of Clinical Oncology, vol. 31, no. 26, pp. 3205-3211, 2013.

[49] K. T. Flaherty, C. Robert, P. Hersey et al., "Improved survival with MEK inhibition in BRAF-mutated melanoma," The New England Journal of Medicine, vol. 367, no. 2, pp. 107-114, 2012.

[50] S. Ugurel, J. Röhmel, P. A. Ascierto et al., "Survival of patients with advanced metastatic melanoma: the impact of novel therapies," European Journal of Cancer, vol. 53, pp. 125-134, 2016.

[51] S. Yun, N. D. Vincelette, M. R. Green, A. E. Wahner Hendrickson, and I. Abraham, "Targeting immune checkpoints 
in unresectable metastatic cutaneous melanoma: a systematic review and meta-analysis of anti-CTLA-4 and anti-PD-1 agents trials," Cancer Medicine, vol. 5, no. 7, pp. 1481-1491, 2016.

[52] S. P. D’Angelo, J. Larkin, J. A. Sosman et al., "Efficacy and safety of nivolumab alone or in combination with ipilimumab in patients with mucosal melanoma: a pooled analysis," Journal of Clinical Oncology, vol. 35, no. 2, pp. 226-235, 2017. 


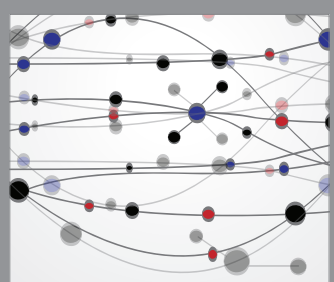

The Scientific World Journal
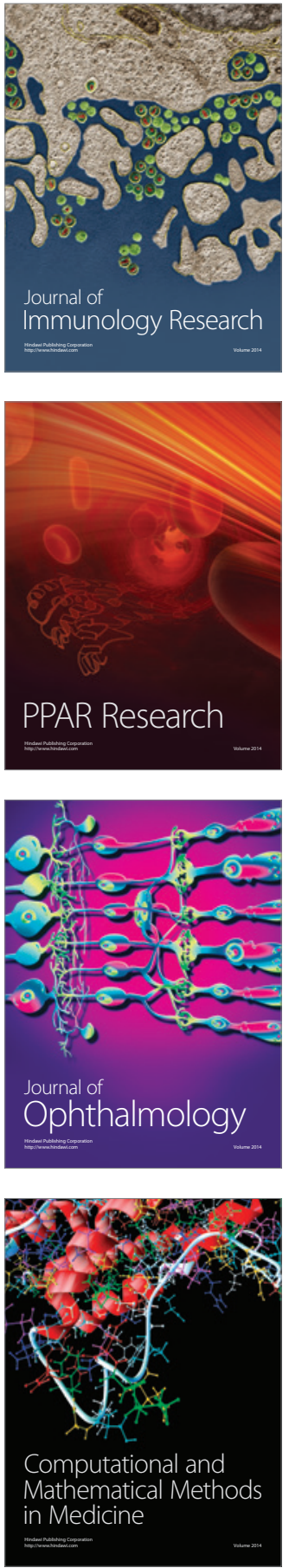

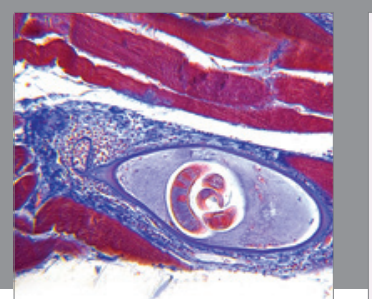

Gastroenterology Research and Practice
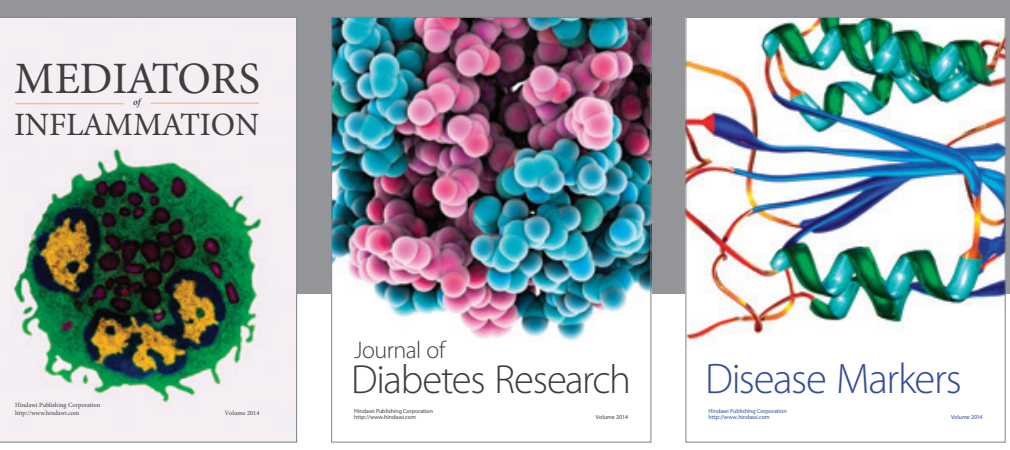

Disease Markers

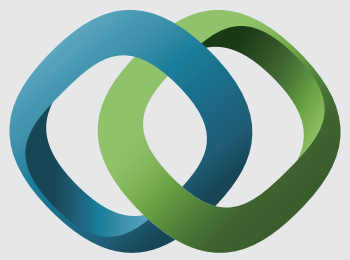

\section{Hindawi}

Submit your manuscripts at

https://www.hindawi.com
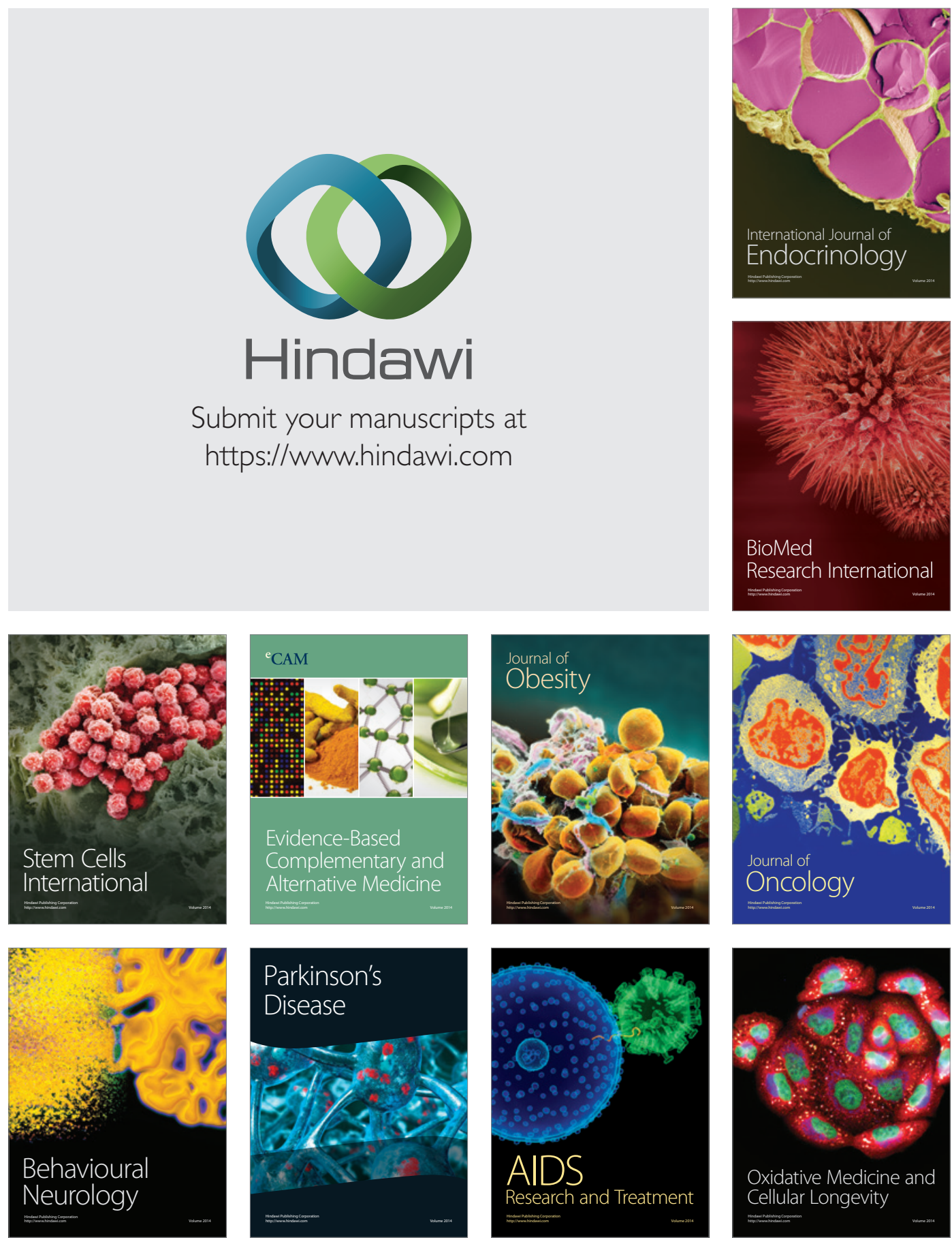